\title{
Necesidades Percibidas en la Atención Hospitalaria Pediátrica a Inmigrantes según la Opinión Profesional: Estudio Cualitativo
}

\section{Perceived Needs and Attitudes in Pediatric Hospital Attention to Immigrants According to the Professional Opinion: Qualitative Study}

\author{
Antonio Fernández-Castillo \\ Universidad de Granada
}

\author{
María José Vílchez-Lara \\ Complejo Hospitalario de Jaén
}

Resumen. En los últimos años la población inmigrante atendida en Unidades y Servicios Hospitalarios pediátricos ha ido en aumento, conforme al incremento en los contingentes de población inmigrante residente en nuestro país. Por el momento no son muchos las investigaciones centradas en las problemáticas y dificultades concretas que la atención a esta población podría plantear. El objetivo de nuestra investigación se centra en conocer, a través de la opinión de los profesionales, variables que pueden incidir en la calidad y satisfacción con dicha atención hospitalaria. Para la consecución de nuestros objetivos de investigación, se ha optado por una metodología cualitativa de tipo fenomenológico. Concretamente se utilizó el procedimiento de entrevistas a informantes clave. Los participantes fueron trabajadores en activo, en Servicios de Pediatría de centros hospitalarios de la Comunidad Autónoma de Andalucía. Las circunstancias que más se relacionan con dificultades en la atención hospitalaria pediátrica incluyen aspectos relacionados con las barreras idiomáticas, seguidos de aspectos socio-económicos, o aspectos psicológicos y de personalidad, durante el proceso de hospitalización. Los resultados obtenidos nos ayudan a comprender dificultades observadas durante la atención a esta población y en estas circunstancias, al tiempo que nos son de utilidad de cara a mejorar la práctica asistencial.

Palabras clave: hospitalización, pediatría, migración internacional, evaluación de necesidades.

Summary. In recent years the increasing of immigrant population attended in hospital pediatric units has been evident, according to the growing number of resident immigrant population in our country. At the moment there is no many investigations centred in problems and concrete difficulties that the attention to this population could present. The objective of our investigation is focused on knowing variables that can impact the quality and satisfaction with this kind of hospital attention, based on the opinion of professionals. For the accomplishment of our investigation objectives, the option has been a qualitative methodology of phenomenology type. To be concrete, interviews to key-informers procedure have

La correspondencia sobre este artículo puede dirigirse al primer autor a la Universidad de Granada. Departamento de Psicología Evolutiva y de la Educación. Facultad de Ciencias de la Educación. Campus de Cartuja s/n 18071 Granada. o a través del correo electrónico:afcastil@ugr.es
Este estudio es parte de otro estudio más amplio realizado en el ámbito de dos proyectos de investigación subvencionados por el Gobierno Autonómico Andaluz. Concretamente por la Consejería de Salud de la Junta de Andalucía. Proyectos con códigos: 0028/06 y 0142/08.

Los autores agradecen a los participantes que desinteresadamente han colaborado y han permitido alcanzar los resultados de esta investigación. A los pedíatras y al personal de enfermería de los hospitales colaboradores. 
been used. The participants were workers of pediatrics services in hospitals in the autonomous region of Andalusia, Spain. The circumstances more related to difficulties in the pediatric hospital attention, in opinion of the professionals, include aspects of idiomatic barriers. This aspect is followed by social and cultural or personality and psychological aspects, during the hospitalization process. The results obtained help us to understand difficulties observed during the attention to this population in pediatric hospitalization circumstances. This is also of great interest trying to improve hospital practice and satisfaction.

Key words: hospitalization, pediatric, international migration, needs Assessment.

\section{Introducción}

El incremento en los últimos años en los contingentes de población inmigrante en nuestro país, se ha evidenciado igualmente en la Comunidad Autónoma de Andalucía, llegando a ser en algunas zonas geográficas concretas muy significativo.

De acuerdo con las cifras del Instituto Nacional de Estadística, Andalucía acoge el $8.1 \%$ de la población inmigrante sobre el total de la misma. En España cerca del $90 \%$ de los inmigrantes se concentran en cinco comunidades autónomas, siendo las tres que más población extranjera acogen: Illes Balears $(21,7 \%)$, Comunitat Valenciana $(17,4 \%)$ y Comunidad de Madrid (16,4\%) (Instituto Nacional de Estadística, 2009). Por su parte la Secretaría de Estado de Inmigración y Emigración, a fecha de 31 de marzo de 2009 reconoce un total de 4.495 .349 de extranjeros con certificado de registro o tarjeta de residencia en vigor, de los que 2.413.144 (el $53,76 \%$ ) son hombres y 2.075 .977 (el $46,24 \%$, mujeres (Secretaría de Estado de Inmigración y Emigración, 2009). Por su parte, el Instituto Nacional de Estadística a tenor de las cifras del padrón municipal, sitúa el número de extranjeros empadronados en 5,6 millones, lo que supone un $12 \%$ sobre el total de la población empadronada (Instituto Nacional de Estadística, 2009). Sin embargo otras fuentes sitúan desde hace tiempo a nuestro país a la cabeza de la Unión Europea en número de inmigrantes, alcanzando ya dicho contingente cifras superiores sobre el total de la población (Etnia Comunicación, 2007). Estas cifras nos ubican por delante de países tradicionalmente acogedores de inmigrantes como Francia, Alemania o Reino Unido. Sin embargo, ni siquiera estos datos podrían reflejar la realidad pues posiblemente no se incluiría en ellos la totalidad de inmigrantes no regularizados que viven en nuestro país.

En relación con el contingente de población infantil de origen inmigrante, las cifras oficiales proceden de dos fuentes primordiales. La Secretaría de Estado de Inmigración indica que en España residen 605.079 extranjeros menores de 16 años (Secretaría de Estado de Inmigración y Emigración, 2009). Por su parte, el Ministerio de Educación y Ciencia señala que el alumnado extranjero en enseñanzas no universitarias en el curso académico 2006-2007 suponía un total de 608.040 alumnos extranjeros (Ministerio de Educación y Ciencia, 2007a; 2007b), pasando en el curso 2007-2008 a 695.190 alumnos, (lo que supone un aumento respecto al curso anterior de más del 14,0\%) (Ministerio de Educación, Política Social y Deporte, 2009). De estas últimas cifras quedan por supuesto fuera los niños no escolarizados y los menores de 3 años.

Aunque no existen datos específicos, el incremento de la atención hospitalaria en el ámbito pediátrico a la población inmigrante parece ser también considerable. De hecho, las cifras de nacimientos y atención en el puerperio a población inmigrante, por ejemplo, superan a la población autóctona en algunas zonas. Recordemos que España se encontraba por debajo del reemplazo generacional y que esta situación ha sido mitigada por la llegada ingente de inmigrantes de los últimos años (Instituto Nacional de Estadística, 2007).

Entre las variables de carácter social que pueden incidir en el nivel de salud pediátrico de la población inmigrante, podríamos destacar la situación socioeconómica de esta población, aspectos de carácter cultural y los aspectos asociados a la migración en sí.

Con respecto al primero de ellos, la situación económica y social, es frecuente que los inmigrantes, al 
menos en un primer momento, se ubiquen en estratos y sectores deprimidos o desfavorecidos en comparación con el grueso de la población autóctona (Molero et al., 2001). Las condiciones laborales y de vivienda son en ocasiones deficitarias, generando todo ello situaciones que pueden estar asociadas a déficit en las condiciones sanitarias. Nos encontramos con frecuentes casos de pobreza y marginalidad, donde la salubridad, la alimentación o incluso la higiene, pueden ser cuestionables.

Por otra parte el impacto o el efecto que diferentes bagajes culturales podrían ejercer sobre el cuidado de la salud es sin duda una variable a tener en cuenta, e incluso el propio concepto de salud- enfermedad puede cambiar de forma significativa entre sociedades y culturas distintas. Si el desconocimiento del lenguaje mutuo en las interacciones entre el personal sanitario y el paciente se incluye en este apartado, estaremos quizá ante un problema que en ocasiones puede estar entre los mayores. La barrera idiomática podría motivar incluso la imposibilidad de comunicación, la dificultad durante las pruebas diagnósticas o incluso de cara al diagnóstico o al cuidado y desempeño de labores sanitarias diversas. Este escenario de asimetría de información y demás particularidades se hace extensivo al ámbito pediátrico en igual o mayor medida que en otras especialidades médicas.

Las circunstancias específicas que se asocian al proceso migratorio generan en multitud de casos contextos familiares y personales complejos y desestructurados (Zarza y Sobrino, 2007). Es frecuente encontrarnos con situaciones de soledad, desorganización familiar, redes sociales y de apoyo disfuncionales o inexistentes, unido a las habituales dificultades de inserción y adaptación a una sociedad desconocida y diferente a la propia (Miranda et al., 2005). A todo esto se une en ocasiones la presencia de alteraciones psicológicas y emocionales tales como ansiedad y estrés durante el proceso de atención sanitaria (Sánchez, 2003; López-Naranjo y FernándezCastillo, 2004; Krohne y Slangen, 2005). Por último, todo ello se ve potencialmente agravado en situaciones de ilegalidad si no se tiene la documentación de permanencia en nuestro país regularizada.

En el momento actual, las instituciones políticas y sanitarias continúan garantizando el acceso a la atención médica pública. Ello no implica que muchos inmigrantes, sobre todo los que llevan poco tiempo en nuestro territorio, tengan dificultades en el acceso a los servicios básicos y especializados de salud, o que desconozcan mínimamente el funcionamiento del sistema sanitario y las posibilidades de utilización. Pero además de estas circunstancias, podríamos destacar los escasos esfuerzos de las instituciones y servicios sanitarios para fomentar la competencia cultural que permita no sólo la accesibilidad, sino incluso una atención más eficiente a este colectivo (Martínez, Martínez y Calzado, 2006).

También hemos de ser conscientes que hablar de población inmigrante en sentido general implica una falacia derivada de posibles generalizaciones. Nos inducirá indudablemente a error comparar personas con orígenes culturales y geográficos muy diferentes como por ejemplo personas procedentes del Caribe, del Magreb o de Europa del Este.

Fruto de estas generalizaciones y de la falta de competencias interculturales, la prestación de servicios a esta población es en ocasiones inadecuada por cuanto pueda resultar poco ajustada o sensible a situaciones específicas. Algunos autores (Martínez, Martínez y Calzado, 2006) han insistido en la necesidad de fomentar la competencia cultural de los profesionales que trabajan con usuarios procedentes de otras zonas geógraficas. Así por ejemplo se entiende imprescindible incrementar la sensibilidad a la diversidad cultural cada vez más presente en nuestro país así como la potenciación del conocimiento en los profesionales y las instituciones sobre aspectos diferenciales de sus propios usuarios. Se ha señalado en esta línea lo adecuado que podría ser el incremento de la concienciación sobre cómo nuestro propio bagaje cultural podría incidir en las relaciones profesionales y de atención al usuario entre otras cuestiones (Martínez, Martínez y Calzado, 2006).

Aunque de un tiempo a esta parte se están desarrollando investigaciones centradas en las circunstancias que dificultan la atención sanitaria a población inmigrante, no son muchos los estudios centrados en el proceso de ingreso hospitalario y menos aún en el caso concreto de la infancia hospitalizada.

El objetivo general de la presente investigación es conocer las necesidades básicas en la atención hos- 
pitalaria pediátrica de la población inmigrante, a través de la opinión de los profesionales sanitarios de centros hospitalarios, y buscar aspectos diferenciales con la población autóctona.

Como objetivos específicos nos planteamos:

A) Detectar los factores psicológicos y contextuales asociados a la atención pediátrica hospitalaria profesional, poniendo especial énfasis en las barreras que dificultan la misma.

B) Explorar la existencia y funcionamiento de recursos institucionales específicos para la atención hospitalaria a esta población.

C) Detectar diferencias en el uso de los recursos sanitarios en función de factores sociales, culturales, origen geográfico, etc., de esta población.

D) Determinar las características del uso de los recursos sanitarios en esta población.

E) Describir las estrategias de cambio en la política sanitaria y en los recursos institucionales propuestas por los profesionales para la mejora de la atención a esta población.

\section{Material y método}

Dados los objetivos de investigación propuestos, nos decantamos por el uso de una metodología cualitativa de tipo fenomenológico (Navarro y Díaz,
1999). Como instrumento para la recogida de la información se usó una entrevista semiestructurada individual y abierta a informantes clave. La muestra de sujetos estuvo compuesta por 15 profesionales sanitarios de servicios de pediatría de hospitales andaluces, concretamente de las provincias de Almería, Granada y Málaga. Algunas de sus características se detallan en la tabla 1.

Como método de muestreo se utilizó el de tipo intencional y en "bola de nieve". De acuerdo con ello algunos de los entrevistados, elegidos por su adecuación a los criterios señalados, sugirieron la participación de otros profesionales. La selección de los participantes se centró en dos perfiles profesionales: facultativos especialistas de área (en adelante FEAs) en pediatría y personal de en enfermería. Tras las 15 entrevistas referidas se observó la saturación del discurso, lo que dio lugar a la finalización de esta fase de la investigación así como al límite del número de informantes-clave participantes. Los criterios de segmentación, y por consiguiente de selección, de los participantes de la muestra incluyeron por tanto dos perfiles profesionales: ser FEA en pediatría o enfermero/a en activo en hospitales de Andalucía Oriental con una atención relevante a población inmigrante, obteniéndose así los criterios de homogeneidad. Igualmente hay que señalar que para la segmentación muestral se tuvieron en cuenta los propios objetivos del proyecto, así como el marco

Tabla 1. Características de los sujetos participantes en la investigación

\begin{tabular}{lcccc}
\hline & Sexo & $\begin{array}{c}\text { Antigüedad } \\
\text { hospitalaria } \\
\text { en Pediatría } \\
\text { En años }\end{array}$ & $\begin{array}{c}\text { Antigüedad Hospitalaria } \\
\text { en Atención Primaria } \\
\text { En años }\end{array}$ & $\begin{array}{c}\text { Localización } \\
\text { del Hospital }\end{array}$ \\
\hline Facultativo especialista en Pediatra & Hombre & 14 & 0 & Urbana \\
Facultativo especialista en Pediatra & Hombre & 4 & 0 & Urbana \\
Facultativo especialista en Pediatra & Mujer & 3 & 0 & Urbana \\
Facultativo especialista en Pediatra & Hombre & 4 & 0 & Rural \\
Facultativo especialista en Pediatra & Mujer & 14 & 7 & Rural \\
Supervisora de Enfermería & Mujer & 7 & 14 & Rural \\
Facultativo especialista en Pediatra & Hombre & 16 & 6 & Urbana \\
Supervisora de Enfermería & Mujer & 20 & 2 & Urbana \\
Supervisor de Enfermería & Hombre & 16 & 2 & Urbana \\
Facultativo especialista en Pediatra & Mujer & 12 & 1 & Urbana \\
Enfermera & Mujer & 9 & 1 & Rural \\
Diplomada en Enfermería & Mujer & 11 & 0 & Rural \\
Enfermero & Hombre & 7 & 1 & Urbana \\
Facultativo especialista en Pediatra & Hombre & 6 & Rural \\
\hline
\end{tabular}


Figura 1. Categorías utilizadas en el análisis de resultados

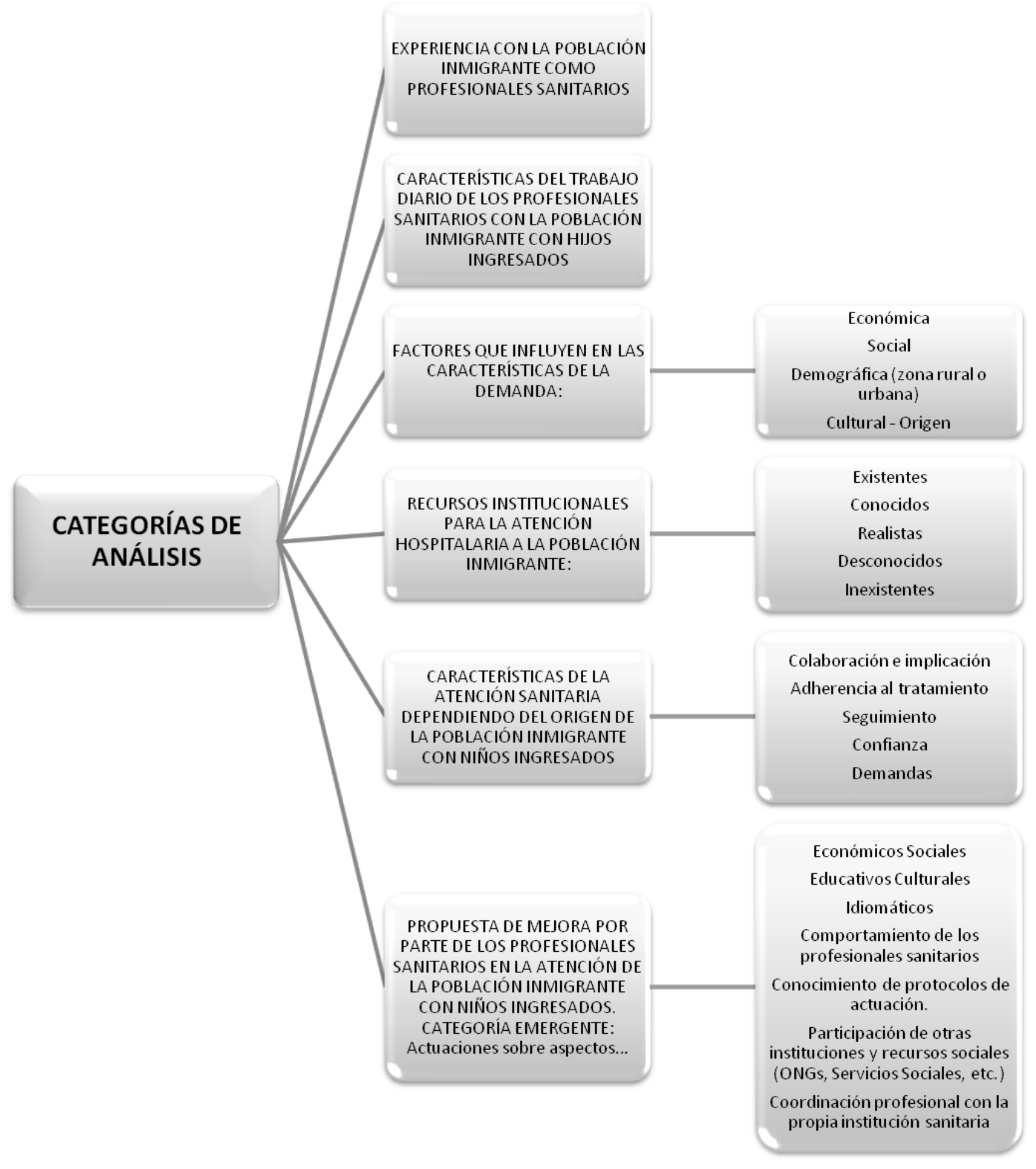

teórico y la bibliografía revisada. Los criterios de heterogeneidad fueron a) el género, b) la experiencia laboral, ser hombre o mujer en el primer caso y tener una experiencia mayor o menor de 10 años en la labor profesional descrita y c) la ubicación rural o urbana del centro hospitalario.

Como criterios de validación se impuso un imperativo acuerdo entre investigadores, consiguiéndose así 
la triangulación. De esa forma se alcanzó un consenso necesario tanto en las pautas de agrupación como sobre todo en los criterios de saturación del discurso o, posteriormente en la obtención de resultados.

Antes de realizar la entrevista, y tras solicitar las oportunas colaboraciones y autorizaciones, los entrevistadores se presentaban, procediendo a informar a los entrevistados de que se iba a proceder a la grabación de la sesión. Se informaba igualmente de los objetivos generales del estudio, se solicitaba el consentimiento en la participación, y se advertía de la posibilidad de abandonar la entrevista si es que así lo consideraban en algún momento. En el mismo sentido se les daba información sobre el carácter confidencial y anónimo de las opiniones vertidas, de la custodia de los datos obtenidos así como de su destrucción tras la finalización de la investigación (Barrio-Cantalejo y Simon-Lorda, 2006; Mercado et al., 2002). Todas las entrevistas fueron desarrolladas por dos entrevistadores con experiencia en investigación cualitativa y registradas en dos aparatos de grabación simultáneamente para después pasar a ser digitalizadas. Las entrevistas tuvieron una duración media global de 40 minutos y aunque eran semiestructuradas, no se limitó a los informantes-clave en sus contestaciones y comentarios, otorgándoles plena libertad en las mismas.

Posteriormente las entrevistas fueron transcritas literalmente para proceder a la categorización de la información, su codificación y la extracción de resultados. En esta fase, y con ayuda del programa Nudist (versión 1.3), se extrajo la información relevante en relación con los objetivos de la investigación. Un análisis semántico fundamentado en las categorías, las cuales se detallan en la figura 1 , fue llevado a cabo en concordancia de nuevo con los propósitos fundamentales del estudio. Dichas categorías se corresponden con el contenido de las preguntas de la entrevista semiestructurada.

\section{Resultados}

En relación con nuestro primer objetivo, los resultados obtenidos delatan la presencia de alteraciones emocionales, fundamentalmente miedo, ansiedad y estrés durante el proceso de hospitalización, unido a una desconfianza latente, tanto en los pacientes y sus padres, como en ocasiones en el propio personal sanitario.

Los problemas de comunicación derivados de la barrera idiomática, a excepción de los inmigrantes latinoamericanos, son una dificultad ampliamente señalada por los profesionales. Esta eventualidad se asocia frecuentemente con dificultades en la atención sanitaria e implica cuando menos una mayor atención hospitalaria, sobre todo si se produce falta de entendimiento de normas de funcionamiento, dificultades en el seguimiento de instrucciones, etc.

Algunos entrevistados han señalado la interacción de las dos circunstancias anteriores: dificultades con el idioma y ansiedad y desconfianza. Esta confluencia se asocia a problemas de comunicación, aislamiento e incluso una ocasional falta de colaboración.

También han sido aspectos referidos problemas en el seguimiento de los casos, el abandono o la no asistencia a citas de revisión, etc., señalándose mayores dificultades en la población de origen inmigrante que en la autóctona. A ello se une una cierta dejadez y abandono en el seguimiento de las instrucciones indicadas por los profesionales, la automedicación o la adherencia al tratamiento: "La adherencia suele ser mala, suele ser bastante mala en general. No sabemos si porque no se enteran bien de lo que se le está diciendo o por qué... no se, una vez que se les deriva, rechazan la continuación. Pensarán que si ya están bien, si ya ha pasado la causa de ingreso, para qué seguir con el seguimiento o el tratamiento... eso cuesta muchas veces..."

Las diferencias culturales han sido señaladas también como relevantes en algunos casos y sobre todo en relación con grupos de inmigrantes específicos. En ocasiones el bagaje cultural puede interferir con los procesos diagnósticos o los tratamientos, aunque esta particularidad es en realidad poco frecuente. Por ejemplo algunos entrevistados han señalado mayor dificultad de comunicación con mujeres magrebíes cuando se trata de un profesional de género masculino, tendencia al uso de la medicina tradicional en algunos inmigrantes sudamericanos o de origen asiático, etc., aunque estas opiniones pueden entenderse como generalizaciones que habría que interpretar con cautela. Se han referido casos en que los padres 
han divergido de la opinión médica, han preferido tratamientos alternativos o no se han ceñido adecuadamente al tratamiento, las revisiones, etc.

Aunque lo más frecuente es que cuando estos problemas se presentan, no sean tanto debidos a diferencias culturales como a aspectos de carácter socioeconómico o a las ya referidas barreras idiomáticas y dificultades de comunicación. Entre estas variables estarían la pobreza, el estar en situaciones de irregularidad, vivir en zonas rurales o alejadas del centro hospitalario o la falta de una red social adecuada para el sostén de la vida familiar durante el ingreso hospitalario, por no hablar de otras circunstancias como los continuos desplazamientos por el territorio nacional en algunos casos o situaciones de marginalidad o deterioro de la estructura familiar en otros. "Hay inmigrantes que están de forma muy temporal en nuestro área e incluso en nuestra región, encontramos a veces personas con las que es muy difícil establecer relación documental, porque precisamente carecen de documentos y por supuesto de historia médica, y aunque se intentan limar esas diferencias ellos lo advierten como una amenaza. Todo ello supone dificultades a la hora de establecer un tratamiento".

También se han referido dificultades asociadas al género, pues en algunos grupos de inmigrantes, es frecuente encontrar un porcentaje mayor de analfabetismo en las mujeres o la dependencia del hombre para tomar decisiones que atañen a la realización de pruebas diagnósticas o tratamientos concretos en los niños ingresados. Esta circunstancia puede incidir ocasionalmente también en la labor profesional.

Respecto a nuestro segundo objetivo y a pesar de que las políticas sanitarias andaluzas garantizan la cobertura a la población inmigrante, todavía se observan barreras en la aproximación y dificultades en el acceso a la atención médica y hospitalaria.

Aunque los recursos institucionales y gubernamentales son suficientes, en ocasiones y en algunos centros se echa en falta recursos personales y profesionales concretos considerados como necesarios. Específicamente se demandan intérpretes, mediadores culturales, etc., existentes en algunos hospitales, pero no en todos.

También se conoce la existencia de recursos para afrontar los problemas de comunicación derivados de las barreras idiomáticas antes comentados. En ese sentido y por ejemplo, existen teléfonos de traducción simultánea, aunque en ocasiones su funcionamiento no es demasiado adecuado o ágil, o folletos idiomáticos y fichas en los más frecuentes idiomas. Estos recursos requieren que el usuario sepa leer, incluso en su lengua materna, lo que no siempre ocurre. Es muy frecuente que los médicos y el personal de enfermería tenga que echar mano en ocasiones de otros inmigrantes ingresados en el centro con mayor conocimiento del castellano para que hagan de interpretes, con la consiguiente pérdida de intimidad o de seguridad en las traducciones, o de voluntarios y personal de Organizaciones No Gubernamentales (ONGs), que no siempre son expertos o profesionales.

En la mayoría de los centros existen protocolos de atención a población inmigrante, aunque no siempre se siguen o se conoce su existencia por parte de los profesionales sanitarios. También en algún caso se ha señalado una cierta ineficacia en la vehiculización y organización de los recursos existentes.

Aparte de estas consideraciones, no existen necesidades médicas específicas de esta población que requieran de mayores recursos o infraestructuras en comparación con la población autóctona.

En relación con el tercero de nuestros objetivos, casi todos los aspectos reseñados son comunes a la mayor parte de la población inmigrante. Algunos de los entrevistados señalaron características diferenciales entre distintos grupos de personas inmigrantes en función de su origen. Así, por ejemplo los inmigrantes de origen magrebí parecen tener una mala adherencia al tratamiento, no practicar demasiada medicina preventiva y presentar mayores dificultades de cara al seguimiento, al igual que la población de origen subsahariano. Los norteafricanos fueron también descritos como más receptivos, pero en ocasiones son los que más dificultades suelen plantear de cara a la instauración de tratamientos y seguimientos. En esta población, además de la barrera idiomática y las diferencias culturales, se destacó en algunos casos la dificultad en la interacción con las madres durante el proceso de hospitalización, pues ellas, aunque se suelen ocupar del cuidado y la atención del hijo durante el ingreso, suelen delegar en sus maridos el contacto con el personal 
sanitario y la toma de decisiones: "Es frecuente que las mujeres magrebies sean reticentes a hablar con nosotros, sí algo más con las enfermeras, pero es el marido o a veces otro hombre de la familia el que pregunta o el que va a tomar las decisiones".

Los inmigrantes procedentes de países del Este fueron descritos como muy consultadores y demandantes de servicios, aunque se les reconoce un gran esfuerzo por comunicarse y entender el idioma. En este sentido los menos exigentes fueron los subsaharianos, quienes en ocasiones incluso tardan demasiado en hacer la demanda de servicios de salud.

Posiblemente los inmigrantes latinoamericanos, sean los que menores dificultades plantean, sobre todo por la inexistencia de barrera idiomática.

La unanimidad casi es total entre los informantesclave en la consideración de que más que factores de carácter geográfico o la cultura de origen del inmigrante, otras variables podrían explicar en mayor medida la mayor parte de dificultades. Entre esas variables estarían el nivel social, económico y cultural, unido a la propia situación de irregularidad en la estancia en nuestro país. En palabras textuales: " $E l$ problema no es tanto el origen racial como el "origen" económico. ... no es lo mismo el hijo de un jugador de baloncesto por ejemplo rumano, que es de origen inmigrante, que el hijo de un rumano que se dedica a la mendicidad y los dos son inmigrantes. Para mi está claro que no es una cuestión racial y si una cuestión económica y social." Estas variables y no tanto el origen del inmigrante, marcan en realidad los aspectos referidos anteriormente.

Por lo que se refiere al cuarto de nuestros objetivos, desde el punto de vista de los profesionales sanitarios entrevistados, no parecen existir demasiadas diferencias en el uso de los recursos hospitalarios cuando comparamos población inmigrante y autóctona. Algunas de las diferencias referidas se relacionan por ejemplo con un mayor uso de las urgencias pediátricas hospitalarias por parte de la población inmigrante, quienes prefieren esta vía de atención médica a los canales habituales a través de la atención primaria: "A veces encontramos familias que son muy consultadoras, sobre todo y muchas veces hiper-consultadores de servicios de urgencias, parece que prefieren ir a urgencias que a su médico de cabecera. Lo mismo es que las urgencias les parecen más seguras o que en primaria les van a poner trabas o problemas".

También existe un consenso entre nuestros entrevistados en relación con dificultades de salud en la población inmigrante, en el sentido de que no se observan patologías diferentes o llamativamente presentes en esta población en comparación con la población no inmigrante.

En un sentido general igualmente, nuestros entrevistados coincidieron en su percepción de una buena satisfacción con la atención sanitaria recibida durante la estancia hospitalaria por parte de la población inmigrante, sobre todo en comparación con la población autóctona. En la misma línea no se han encontrado diferencias significativas entre centros hospitalarios ubicados en zonas rurales o urbanas. En lugar de esta circunstancia sí se detecta una mayor dotación de determinados recursos si la población inmigrante que reside en el área de atención es más o menos numerosa. Entre estos recursos destaca quizá la presencia de determinados profesionales para la eliminación de barreras de comunicación.

Con respecto al último de nuestros objetivos, existen una serie de propuestas claras señaladas por los informantes clave. La primera de ellas es la importancia incuestionable de la presencia en los centros hospitalarios de intérpretes y mediadores culturales, los cuales representan una de las medidas más eficaces para romper las barreras idiomáticas y culturales cuando estas se presentan. Su labor representa una forma directa de eliminar obstáculos en la comunicación e interacción con inmigrantes.

En algún caso se ha destacado también lo oportuno que resultaría contar con mayores recursos formativos para los profesionales que han de trabajar con porcentajes significativos de población inmigrante, explícitamente se señaló el interés en contar con cursos, foros y actividades profesionales dirigidas en ese sentido. Según diversas opiniones, no son demasiados los esfuerzos formativos dirigidos a los profesionales sanitarios en aspectos referidos a la atención a inmigrantes. $\mathrm{Y}$ esto se constituye en una propuesta de mejora clara. En esta línea podrían ser de alto interés iniciativas de formación en competencias culturales e interculturales, de acuerdo con diversos autores (Martínez, Martínez y Calzado, 2006).

\footnotetext{
Copyright 2009 by the Colegio Oficial de Psicólogos de Madrid
} ISSN: 1132-0559 
También se ha hecho referencia por algunos entrevistados a dificultades o poca atención a la relación entre profesionales, a dificultades en el flujo de información, la inexistencia de una atención integral y a la interacción de los profesionales con la propia institución sanitaria para la creación y planificación de protocolos y estrategias de intervención específicas.

Por último se constata la necesaria incorporación y el trabajo con otras instituciones que pueden paliar algunos de los problemas de carácter social y económico que presenta la población inmigrante. Se señalan por ejemplo servicios sociales comunitarios y educativos $\mathrm{u}$ organizaciones no gubernamentales que pueden desempeñar un papel primordial en ese sentido. La propuesta específica de mejora de los canales de comunicación e interacción profesional con estos servicios y entidades fue igualmente una propuesta específica de mejora.

\section{Discusión}

En el estudio llevado a cabo nos planteábamos conocer aspectos diferenciales inherentes y dificultades específicas en la atención hospitalaria pediátrica a población de origen inmigrante. Pensamos que este tema resulta de interés a tenor de los contingentes tan elevados de población extranjera que en los últimos años se dan en nuestro país, y que lo ubican como el de mayor porcentaje de población inmigrante de nuestro entorno.

Nuestra perspectiva de estudio se basa en la opinión de profesionales con constatada experiencia que participaron como informantes-clave. Debido a este planteamiento metodológico es sin lugar a dudas importante recordar que los resultados obtenidos no son más que las opiniones de los profesionales entrevistados, por lo que son en definitiva matizables y pueden no adecuarse a la realidad general. Dichas opiniones, aún proviniendo de expertos, han de relativizarse y como mínimo circunscribirse al escenario en que han sido obtenidas. Esta eventualidad ha de reconocerse como una limitación de este tipo de estudios cualitativos.

Entre los aspectos psicológicos señalados, han sido destacadas fundamentalmente la presencia de alteraciones emocionales tales como miedo, estrés y ansiedad en la población inmigrante. $\mathrm{Y}$ aunque dichos estados no son exclusivos de esta población en las circunstancias de hospitalización infantil, (Fernández-Castillo y López-Naranjo, 2006; Ortigosa y Méndez, 2000) sin lugar a dudas merecerían de una mayor atención por parte de los investigadores.

El desconocimiento del funcionamiento de los estamentos sociales y sanitarios, la falta de medios económicos o de apoyo familiar y social en algunos casos suelen ser factores que incrementan las barreras de acceso a los servicios hospitalarios. Ejemplos de ello son las dificultades para hacer desplazamientos hasta los centros sanitarios si están distantes del domicilio familiar, para pernoctar en la ciudad donde se encuentra el hospital si el domicilio familiar está distante o el tener que abandonar obligaciones laborales y familiares. Todos ellos pueden ser obstáculos en ocasiones insalvables para algunas familias (Yantzi et al., 2001). En estos casos la adherencia al tratamiento, que puede ser costosa, o la asistencia a citas con los profesionales, puede verse dificultada (Arellano-Millán y Fernández-Franco, 2005). Para algunas madres sin dominio del idioma e incluso analfabetas, con pocos recursos económicos y sociales en muchos casos, manejarse por una sociedad nueva y diferente hasta llegar al centro médico, se convierte en una dificultad.

En relación con recursos institucionales y medios, un aspecto frecuentemente señalado ha sido la necesaria presencia de intérpretes que puedan evitar la aparición de barreras idiomáticas, así como la incorporación de mediadores culturales, que permitan que se den sin problemas los cauces mínimos de comunicación. Todavía nos encontramos con centros hospitalarios en zonas geográficas con presencia significativa de población inmigrante, donde estos profesionales no existen en la plantilla y han de ser suplantados por voluntarios o profesionales de otras entidades u ONGs, o por la participación de otros pacientes que en ese momento están siendo atendidos en el mismo centro hospitalario (Hughes, 2001; Martín-Agudo et al., 2007).

Los profesionales no indican diferencias significativas en cuanto a enfermedades o procesos patológicos concretos en la población inmigrante cuando 
se compara con la población autóctona. Dichos resultados, que concuerdan con otras investigaciones (Sánchez, 2003; Cuadrado et al., 2004), tienen sentido si tenemos en cuenta que la población de origen inmigrante es en general joven y que sobre todo la segunda generación, presenta perfiles sanitarios equiparables a los niños no de origen inmigrante.

Por lo que respecta diferencias en el uso de recursos sanitarios, y como hemos comentado, la colaboración durante la hospitalización es buena, aunque el seguimiento posterior en ocasiones no lo es tanto. Es frecuente que se haga caso omiso de los consejos y diagnósticos, la falta de adherencia al tratamiento o el abandono y la no asistencia a citas posteriores, etc., lo que concuerda con otros estudios (Salazar et al., 2003; López de Dicastillo y Cheung, 2004). Estas circunstancias habría que valorarlas nuevamente en el marco de la situación social y económica y cultural así como a tenor del funcionamiento de barreras de acceso particulares para un gran contingente de población inmigrante.

También en relación con el uso de los recursos sanitarios, destaca el uso prioritario y en ocasiones injustificado de las urgencias sanitarias por parte de la población inmigrante, el cual ha sido señalado también por otros autores (Cots et al., 2007) y merecería atención por parte de estudios futuros. Los resultados encontrados sugieren la potenciación y puesta en funcionamiento de medidas que racionalicen el uso de las urgencias hospitalarias, lo que en el caso de la población inmigrante incluiría el acceso a los conocimientos sobre el funcionamiento de los sistemas sanitarios, las opciones y los cauces adecuados para llevar a cabo las demandas concretas o el uso prioritario de la atención primaria. Todo ello en el marco de un esfuerzo de educación sanitaria y para la salud.

En relación con el trato y la atención recibida durante el proceso de hospitalización, los niveles de satisfacción percibida por el usuario son bastante buenos desde el punto de vista de los profesionales.

A pesar de los esfuerzos llevados a cabo en los últimos años en las políticas sanitarias, se debería continuar fomentando la formación de los profesionales no sólo en el conocimiento de las características culturales de los principales grupos de población inmigrante presentes en nuestro territorio, sino también en habilidades y competencias de interacción intercultural que puedan potenciar la eficacia de la prestación de servicios a esta población (Martínez, Martínez y Calzado, 2006).

Para concluir, señalar que todas las posibles dificultades que consideremos, pueden verse agravadas siempre en personas con bajo nivel socio-económico y cultural, como es el caso de un buen contingente de esta población inmigrante por motivos económicos (Valtueña, 2000). Y en cualquier caso, la no regularización de la permanencia será sin lugar a dudas un problema añadido y una variable a tener en cuenta.

\section{Referencias}

Arellano-Millán, M. J. y Fernández-Franco, L. (2005). La inserción social de las inmigrantes latinoamericanas en España: migraciones laborales y géneros. Madrid: Universidad Complutense de Madrid.

Barrio-Cantalejo, I. M. y Simon-Lorda, P. (2006). Problemas éticos de la investigación cualitativa. Medicina Clínica, 126, 418-23.

Cots, F., Castells, X., García, O., Riu, M., Felipe, A. y Vall, O. (2007). Impact of immigration on the cost of emergency visits in Barcelona (Spain). BMC Health Serv Res, 7. [Consultado 11-102007]: Disponible en: http://www.biomedcentral.com/content/pdf/1472-6963-7-9.pdf

Cuadrado, S. P., Avalora, N. M., Sánchez, A. R., Fernández, Y. S., Alonso, C. R. P. y Bertolo, J. D. (2004). Characteristics of immigrant women and their neonates. Anuario Pediátrico, 60, 3-8.

Etnia Comunicación. (2007). II Anuario de la Comunicación del Inmigrante en España. [Consultado 24-10-2007]: Disponible en http://www.etniacomunicacion.com/pdf/ndpanua rioII.pdf

Fernández-Castillo, A. y López-Naranjo, I. (2006). Transmisión de emociones, miedo y estrés infantil por hospitalización. Internacional Journal of Clinical and Health Psychology, 6, 631-645. [Consultado 9-10-2007]: Disponible en: http:// www.aepc.es/ijchp/articulos_pdf/ijchp-196.pdf 
Hughes, B. M. (2001). Psychology, hospitalization, and some thoughts on medical training. European Journal of Psychotherapy, Counselling, and Health, 4, 7-26.

Instituto Nacional de Estadística. (1998). Evolución de la Fecundidad en España 1970-1994. Madrid: Instituto Nacional de Estadística. [Consultado 810-2007]: Disponible en: http://www.ine.es/prensa/np079.htm? $L=0$

Instituto Nacional de Estadística. (2009). Avance del padrón municipal a 1 de enero de 2009. Madrid: Instituto Nacional de Estadística. [Consultado 1706-2009]: Disponible en: http://www.ine.es/prensa/np551.pdf

Krohne, H. W. y Slangen, K. E. (2005). Influence of Social Support on Adaptation to Surgery. Health Psychology, 24, 101-105.

López de Dicastillo, O. y Cheung, P. (2004). La enfermería infantil y los cuidados centrados en la familia. Enfermería Clínica, 14, 83-92.

López-Naranjo, I. y Fernández-Castillo, A. (2004). Aspectos psicosociales y evolutivos en la hospitalización infantil. Revista de Psicología Social Aplicada, 14, 5-27.

Martín-Agudo, M. Y., Sierra-Quesada, J. M., Jiménez-Martín, J. M. y Escudero-Espinosa, C. (2007). Expectativas y necesidades detectadas en profesionales de organizaciones provinciales que trabajan en acción intersectorial en salud. Revista Especializada Salud Pública, 81, 43-52.

Martínez, M.F., Martínez, J. y Calzado, V. (2006). La competencia cultural como referente de la diversidad humana en la prestación de servicios y la intervención social. Intervención Psicosocial, 15, 331-350.

Mercado, J., Gastaldo, D. y Calderón, C. (2002). La investigación cualitativa en salud en Iberoamérica. Métodos, análisis y ética. Guadalajara: Universidad de Guadalajara.

Ministerio de Educación y Ciencia. (2007a). Datos estadísticos de alumnado extranjero. Madrid. [Consultado 14-11-2007]: Disponible en: http://www.mecd.es/mecd/jsp/plantilla.jsp?id=31 0\&area $=$ estadisticas \&contenido=/estadisticas $/$ e ducativas/eenu/avances/Curso06-07/avan ces.html

Ministerio de Educación y Ciencia. (2007b). Series de alumnado extranjero. Madrid. [Consultado 1211-2007]: Disponible en: http://www.mecd.es/ mecd/estadisticas/educativas/eenu/avances/Curs o06-07/SeriesExt.pdf

Ministerio de Educación, Política Social y Deporte. (2009). Datos y cifras. Curso escolar 2008/2009. Madrid: Secretaría General Técnica. [Consultado 22-06-2009]: Disponible en: http://www.educacion.es/mecd/estadisticas/educativas/dcce/DATO S_Y_CIFRAS_WEB.pdf

Miranda, J., Siddique, J., Der-Martirosian, C. y Belin, T. R. (2005). Depresion among latina immigrant mothers separated from their children. Psychiatr Serv, 56, 717-720.

Molero, F., Navas, M. y Morales, J. F. (2001). Inmigración, prejuicio y exclusión social: reflexiones en torno a algunos datos de la realidad española. International Journal of Psychology and Psychological Therapy, 1, 11-32.

Navarro, P. y Díaz, C. (1999). Análisis de contenido. En: Delgado, J. M., Gutiérrez, J., eds. Métodos y técnicas cualitativas de investigación en ciencias sociales. Madrid: Síntesis, 177-224.

Ortigosa, J. M. y Méndez, F. X. (2000). Hospitalización infantil. Repercusiones psicológicas. Madrid: Biblioteca Nueva.

Salazar, A., Navarro-Calderón, E., Abad, I., Alberola, V., Almela, F., Borrás, R., et al. (2003). Diagnóstico al alta hospitalaria de las personas inmigrantes en la ciudad de Valencia (20012002). Revista Española de Salud Pública, 77, 713-723.

Sánchez, T. (2003). Inmigración y síndrome de estrés post-traumático. Revista Española de Pediatría, 59, 367-369.

Secretaría de Estado de Inmigración y Emigración. (2009). Extranjeros con certificado de registro o tarjeta de residencia en vigor y Extranjeros con autorización de estancia por estudios en vigor a 31 de marzo de 2009. Madrid: Ministerio de Trabajo e Inmigración. [Consultado 18-06-2009]: Disponible en: http://extranjeros.mtin.es/es/ InformacionEstadistica/Informes/Extranjeros 31 Marzo2009/Archivos/Informe_Trimestral_31-032009.pdf

Valtueña, O. (2000). Medidas para proteger y promover la salud de los inmigrantes en España. 
Migraciones, 8, 231-250.

Yantzi, N., Rosenberg, M. W., Burke, S. O. y Harrison, M. B. (2001). The impacts of distance to hospital on families with a child with a chronic condition. Soc Sci Med, 52, 1777-1791.
Zarza, M. J. y Sobrino, M. I. (2007). Estrés de adaptación sociocultural en inmigrantes latinoamericanos residentes en Estados Unidos vs. España: Una revisión bibliográfica. Anales de Psicología, $23,72-84$.

Manuscrito recibido: 22/06/2009

Revisión recibida: 21/07/2009

Manuscrito aceptado: 27/08/2009 